\title{
Are Opioids Overprescribed to Pediatric Neurosurgery Patients? A Prospective Study on Prescribing Practices and Patient Use
}

\author{
Leah Burroughs ${ }^{1}$, Denise Ash ${ }^{2}$, Laurie Ackerman ${ }^{1,2}$ \\ ${ }^{1}$ Indiana University School of Medicine; ${ }^{2}$ Department of Neurosurgery, Riley Hospital for \\ Children at Indiana University Health
}

Background and Hypothesis: The opioid crisis continues to worsen in the United States with opioid overdose deaths reaching record highs in 2020. While a large body of literature exists surrounding the risks of opioids in adults, opioids also pose unique risks to pediatric patients, including accidental ingestion, nonmedical use, and acute cerebellitis causing death. Opioid medications prescribed in the medical setting are often an unwitting source of excess opioids, with half of pediatric overdoses in those under 2 years of age. Although legislative efforts have significantly limited opioid prescribing, recent studies suggest these medications may still be overprescribed. We hypothesized opioid medications are overprescribed to pediatric neurosurgery patients upon hospital discharge.

Methods: Pediatric patients undergoing neurosurgical procedures at Riley Hospital for Children were identified prospectively. Surgery type, length of stay, and inpatient use of opioid medications were collected. Patients prescribed an opioid medication upon hospital discharge were contacted 7 days after discharge and asked to report the number of doses of opioid medication used.

Results: Thirty patients were successfully contacted 7 days after hospital discharge. Patients underwent a variety of cranial and spinal procedures and the mean length of hospital stay was 3.9 days. An average of 24.9 doses of opioid medication were prescribed at hospital discharge, while an average of 3.8 doses were used by patients in the 7 days following hospital discharge. Twelve patients $(40 \%)$ had used zero doses of the prescribed opioid medication at 7 -day followup.

Conclusions: Pediatric neurosurgery patients used only $15.3 \%$ of prescribed opioids in 7 days after hospital discharge. This creates an excess of leftover opioid medication that may increase the risk of accidental ingestion and misuse. The present study highlights the need for educational initiatives for providers to minimize excess opioids prescribed and for parents to safely dispose of leftover opioid medication. 